\title{
Zur Strukturanalyse von primären Umwelten
}

\author{
Peter Kappelhoff \\ Institut für Soziologie der Universität Kiel \\ Olshausenstraße $40 \rightarrow 60$, D-2300 Kiel
}

$\mathrm{Z}$ u s a m m e $\mathrm{n}$ f a s s u $\mathrm{ng}$ : Das einfache Zweiebenenmodell beschreibt soziale Struktur, indem es Einheiten der Individualebene denen der Aggregatebene zuordnet und dann die Beziehungen zwischen den beiden Ebenen analysiert. Hier schlagen wir ein erweitertes Dreiebenenmodell vor, das als zusätzliche Tbene die der primären Umwelten als intervenierende Variable enthält. Wir geben dann einen Überblick über verschiedene Methoden zur Cliquenidentifikation in Hinblick auf ihre Anwendbarkeit zur Zerlegung großer Netzwerke in primäre Umwelten und schlagen eine neue Zerlegungsstrategie in sog. Zonen relativer Verdichtung ( $\mathrm{ZrVs}$ ) vor. Nach.einer Metrisierung der internen Struktur dieser ZrVs geben wir verschiedene Indizes an, die die interne Struktur der ZrVs und die Homogenität von Individualmerkmalen in Bezug auf diese Struktur beschreiben.

\section{Zur Problemstellung}

\section{a) Mehrebenenanalyse als ,,social system analysis"}

Die traditionelle Umfrageforschung in den Sozialwissenschaften betrachtet das Individuum losgelöst vom sozialen Kontext und ist daher nicht in der Lage, Aussagen über soziale Strukturen - verstanden als ein relativ dauerhaftes Gefüge von sozialen Beziehungen zwischen Positionen - empirisch zu testen. Untersucht wird vielmehr die Struktur von Merkmalskonstellationen bei Individuen. Mehrebenenanalyse kann verstanden werden als der Versuch einer ,social system analysis" (Riley, 1963) in dem Sinne, daß die verschiedenen Ebenen eines Sozialsystems zueinander in Beziehung gesetzt werden und damit sowohl eine Individualanalyse unter Einbeziehung des sozialen Kontextes als auch eine Gruppenanalyse unter Einbeziehung der die Gruppen konstituierenden Einheiten der niederen Ebene (im Normalfall Individuen) möglich gemacht wird. Die Untersuchung dieser Interdependenzen zwischen den Ebenen ist der Kern der Mehrebenenanalyse.

So verstanden ist Mehrebenenanalyse immer relationale Analyse, da sie von sozialem Handeln ${ }^{1}$ in einer sozialen Beziehung als der Grundeinheit sozialwissenschaftlicher Analyse ausgeht. Kehrt man den Schluß um, so ergibt sich, daß jede sozialwissenschaftliche Analyse Strukturanalyse in

1 Der durch den Begriff des sozialen Handelns gegebene Hinweis auf den Prozeßcharakter des Sozialen solite sich in dynamischen Mehrebenenmodellen widerspiegeln, die in der Lage sind, auch strukturelle Veränderungen im Zeitablauf zu erfassen und zu analysieren. der oben skizzierten Form sein sollte, oder, wie Coleman formuliert: , ... it is precisely because there is some varying structure of relations between individuals that sociology has a reason for existence. Sociology would otherwise be nothing more than ,aggregate psychology', as thermodynamics is an , aggregate statistical mechanics", and would be concerned only with distributions of motives, needs, beliefs, and so on." (1964, S. 88). Damit liegt zugleich auch der Maßstab fest, an dem sich die verschiedenen Mehrebenenmodelle messen lassen müssen nämlich der Grad, zu dem sie relationale Strukturanalyse möglich machen. Im folgenden sollen einige Ansätze zur Mehrebenenanalyse unter diesem Gesichtspunkt vorgestellt und darauf aufbauend ein formales Dreiebenenmodell entwikkelt werden.

\section{b) Das klassische Zweiebenenmodell}

In seiner einfachsten Form kommt der Grundgedanke der Mehrebenenanalyse in dem klassischen Zweiebenenmodell zum Ausdruck. Die Beschreibung der Sozialstruktur liegt in der Zuordnung der Einheiten der Individualebene $z u$ denen der Gruppenebene; die Einheiten der Gruppenebene können als Äquivalenzklassen von Einheiten der Individualebene verstanden werden. Dem entspricht die Möglichkeit, Gruppenmerkmale durch Aggregation von Individualmerkmalen in Form von Durchschnitten, Prozentsätzen, Varianzen oder anderen die Verteilung der Individualmerkmale charakterisierenden Maßzahlen zu gewinnen. Als Beispiel kann die Arbeit von Michael (1961) angeführt werden, in der der Einfluß des „High School-Klimas" auf die Collegabsicht der Schüler untersucht wird. Das durch Aggregation gewonnene Merkmal „,Status der Schule“ wird 
allen Schülern der betreffenden Schule in gleicher Weise als Kontextmerkmal zugeordnet und auf diese Weise eine Analyse der Collegeabsicht unter Einbeziehung des sozialen Milieus möglich gemacht. Allerdings verzichtet dieser Forschungsansatz auf eine weitere Differenzierung der sozialen Position der Schüler innerhalb des umfassenderen Kontextes Schule etwa durch Erhebung von Freundschaftsbeziehungen zwischen den Schülern. Beschrieben wird also nicht die Gruppenstruktur, was eine Berücksichtigung von Beziehungen zwischen den Gruppenmitgliedern voraussetzen würde, sondern die Verteilung eines Merkmals in Bezug auf die Gruppenmitglieder.

Die Verbindung von Individual- und Systemebene im Zweiebenenmodell und die dadurch ermöglichte Analyse der wechselseitigen Beziehungen zwischen den Ebenen sind also vom Ansatz her auf Fragestellungen beschränkt, die eventuell vermittelnde Instanzen zwischen den beiden Ebenen vernachlässigen. Campbell und Alexander kommen zu einer genaueren Analyse des von Michael untersuchten Kontexteffektes, indem sie die ,,position of the individual within the social structure - defined in terms of his specific relationships to other members of the collectivity " als intervenierende Variable einführen, denn ,the actor responds to that segment of the total system which, for him, is perceptually important and salient; rarely does he (inter-) act with reference to the system as a whole" (1965: 284). Die übliche Kontextanalyse wird also durch eine Relationalanalyse ergänzt, indem neben dem Einfluß des „High School-Klimas“ auch der der Schulfreunde - gemessen als deren durchschnittlicher Status - berücksichtigt wird ${ }^{2}$. Vom Ansatz her liegt also ein Dreiebenenmodell vor, wobei aber die Einheiten der zweiten Ebene - in diesem Falle die Freundschaftsgruppen der

2 Wie erwartet finden sich positive Korrelationen sowohl zwischen dem durchschnittlichen Status der Schüler einer Schule (High School-Klima) und der Collegeabsicht der Schüler als auch zwischen dem durchschnittlichen Status der Freunde und der Collegeabsicht der Schüler. Entscheidend für die Deutung des Kontexteffektes ist es nun, daß die erste Korrelation verschwindet, wenn man den Status der Freunde konstant hält, während die zweite Korrelation auch bei Konstanthalten des High School-Klimas erhalten bleibt. Die Wirkung des weiteren Kontextes Schule scheint also in diesem Falle völlig durch den engeren Kontext Freundschaftsgruppe vermittelt zu werden.
Schüler - nicht explizit definiert werden. Dies würde zusätzlich ein Zerlegen des Netzwerkes der Freundschaftsbeziehungen in Cliquen erfordern - eine Aufgabe, die sowohl Kriterien für eine Abgrenzung der Cliquen untereinander als auch einen Algorithmus zur Bestimmung der Cliquen auf der Grundlage dieser Kriterien voraussetzen würde. Die Darstellung einer solchen Prozedur ist das Hauptziel dieses Beitrags.

\section{c) Ein Dreiebenenmodell und mögliche Strategien zur Zerlegung von Kontexten}

Generell erfordert das Zwei-Stufen-Modell von Campbell und Alexander ,a specification of the conditions under which certain structural variables produce these relevant characteristics of an individual's social environment" und ,then use the characteristics of their significant others to predict their behaviors" (S. 288). Dies läuft auf die Forderung nach einer Darstellung von primärer und sekundärer Umwelt im Rahmen eines Dreiebenenmodells hinaus, in dem dann untersucht werden kann, inwieweit ein Kontext höherer Ordnung die Einheiten der niederen Ebenen beeinflußt oder gar determiniert. In dem Begriff des sozialen Feldes versucht Wössner (1969), Ansätze zur Erklärung von sozialem Handeln unter strukturellen Gesichtspunkten zu systematisieren. Danach ist das soziale Feld bestimmt als Konfiguration von Interdependenzen der folgenden drei Faktoren:

„1. Individuelle Faktoren, a.h. Persönlichkeitsfaktoren und Prinzipien der Persönlichkeitsstruktur

2. Faktoren der primären Beziehungen oder auch der informellen Gruppen

3. Faktoren der formalen Organisation und Faktoren der Sekundärbeziehungen." (S. 24)

Mehrebenenanalyse kann dann verstanden werden als Instrument zur Analyse der Wechselwirkungen zwischen den so konzipierten Ebenen der sozialen Realität.

$\mathrm{Zu}$ einer ähnlichen Einschätzung kommt Scheuch in einem Handbuchartikel über Entwicklungsrichtungen bei der Analyse sozialwissenschaftlicher Daten: „Mehrebenen-Analysen sind besonders geeignet, die Beziehung zwischen ,subjektiven" und ,objektiven "Aspekten der sozialen Existenz aufzuzeigen. Einer der möglichen 
Ansätze ist, den Kontext (Gruppe, Bezirk, Organisation) als objektive ,Umgebung' (im Sinne der Verhaltenslehre Uexkülls) zu deuten, auf welche die Individualeinheiten unterschiedlich reagieren. Diese verschiedenen Reaktionen wiederum können als ,Optionen" verstanden werden, als unterschiedliche Präferenzen und Gewohnheiten, durch welche die objektive Umgebung erst subjektiv relevant wird. Verdichten sich je nach Soziallage innerhalb des gleichen Kontextes die Verhaltensweisen zu typischen Reaktionen, so können diese Individualmerkmale als Indikatoren für subjektiv bedeutsame ,Umwelten" (wiederum im Sinne von Uexküll) gedeutet werden ... Dieses Vorgehen, das man als Optionsanalyse bezeichnen könnte, zeigt Art und Grad der Unterschiede zwischen verschiedenen Umwelten innerhalb des gleichen Kontextes auf; und diese Unterschiede sind Indiz für eine der wichtigsten Eigenschaften einer Sozialstruktur: Rigidität bzw. Schwellenwerte, von denen ab die (objektive) Umgebung unmittelbar verhaltensbegrenzend wirkt"“ (1967:676).

Das Problem der Bestimmung der primären Umwelten, d.h. der Definition der Einheiten der zweiten Ebene, wird von Campbell und Alexander in ihrer Untersuchung dadurch umgangen, daß sie eine Zweiebenenanalyse mit einer Relationalanalyse verbinden. Analyseeinheiten für die gesamte Untersuchung sind die Schüler und daher genügt es, eine relationale Charakterisierung von deren Position im Netzwerk der Freundschaftsbeziehungen vorzunehmen und so den für ihre Fragestellung relevanten Aspekt der Einbettung in die primäre Umwelt zu erfassen.

Offenbar reicht eine solche Vorgehensweise nicht aus, wenn Aussagen über die primären Umwelten selbst empirisch getestet werden sollen - was an Hand des erhobenen Datenmaterials durchaus möglich wäre. Erforderlich ist dazu vielmehr die Zerlegung des umfassenderen Kontextes Schule in primäre Umwelten.

Geht man davon aus, daß eine möglichst große interne Übereinstimmung in Bezug auf (relatio. nal definierte) Positionen im umfassenderen Kontext und in Bezug auf relevante (Individual-) Merkmale erreicht werden soll, so bietet sich eine Strategie der Zerlegung auf Grund von Kriterien zur Minimierung der internen Variabilität an. Eine solche Vorgehensweise schlägt Hummell mit der Zerlegung von Kontexten als Be- einflussungsstrukturen nach dem Prinzip maximaler Vollständigkeit (oder Verbundenheit) und Homogenität vor (1972:147). Das Ziel scheint dabei in erster Linie die theoretische Analyse von Bedingungen zu sein, unter denen ein Kontextmerkmal allen Mitgliedern des Kontextes in gleicher Weise zugeschrieben werden kann (im folgenden Kontextmerkmal im weiteren Sinne genannt), ohne interne Differenzierungen in der Struktur des Kontextes berücksichtigen zu müssen. Dies erfordert einmal eine vollständige Kommunikations- bzw. Interaktionsstruktur und zum anderen Übereinstimmung in für die untersuchten Beeinflussungsprozesse relevanten Individualmerkmalen.

Eine konsequente Anwendung dieses Prinzips ergäbe die Zerlegung einer Beeinflussungsstruktur in eine Hierarchie von jeweils vollständigen und homogenen Kontexten und die Anzahl der benötigten Ebenen wäre ein Indikator für die Komplexität des Systems in Hinblick auf die berücksichtigten Merkmale und Interaktionsstrukturen. Insbesondere wären die so erhaltenen primären Umwelten per definitione homogen und vollständig. Dies würde z.B. für eine Freundschaftsgruppe mit nicht vollständiger Struktur und eventuell vorhandenen Inhomogenitäten eine weitere Zerlegung in homogene und vollständige Teilstrukturen bedeuten. Struktur- und Homogenitätsmaße, die die interne Struktur der Freundschaftsgruppe beschreiben, ergäben sich dann durch Analyse der Beziehungen dieser Teilgruppen zueinander im Rahmen der Freundschaftsgruppe.

\section{Die Strategie der Zerlegung nach dem Prinzip} maximaler Vollständigkeit und Homogenität liefert damit zwar die theoretischen Voraussetzungen für eine exakte Strukturanalyse, macht aber zugleich wegen der Komplexität der zu berücksichtigenden relationalen Verflechtungen eine praktisch kaum zu bewältigende Fülle von Relationalanalysen notwendig. Als Alternative bietet sich an, auf die Äquivalenz der Einheiten der niederen Ebene in Bezug auf die durch sie gebildete Einheit der höheren Ebene zu verzichten, also auch Kontexte zuzulassen, die nicht vollständig und homogen sind. Damit ergibt sich dann zusätzlich die Aufgabe, die interne Struktur dieser Einheiten zu erfassen, indem die unterschiedlichen Positionen der Einheiten der niederen Ebene innerhalb der nächsthöheren Ein- 
heit durch Struktur- und Homogenitätsindizes beschrieben werden. Diese Indizes sollen Kontextmerkmale im engeren Sinne genannt werden, da sie zwar auf den gleichen Kontext bezogen sind, die Mitglieder dieses Kontextes aber in unterschiedlicher Weise charakterisieren. Im folgenden soll ein Vorschlag für eine solche Vorgehensweise gemacht werden.

\section{d) Ein formalisiertes Mehrebenenmodell}

Immer dann, wenn Strukturen eines gewissen Komplexitätsgrades abgebildet werden sollen, empfiehlt sich eine formale Darstellung in einer mathematischen Symbolsprache. Die dazu hier verwandte Sprache ist die der Prädikatenlogik erster Stufe, ergänzt durch einige in der Mengenlehre übliche Schreibweisen. $\mathrm{n}$ bezeichne die Anzahl der Ebenen des Modells und $x^{i}, y^{i}, \ldots$ $(i=1, \ldots, n)$ seien Variablennamen für die Elemente der einzelnen Ebenen. Die Beziehungen der Einheiten der verschiedenen Ebenen zueinander (,,vertikale" Struktur) werden durch die $\epsilon$-Relation dargestellt: $x^{i} \in x^{i+1}$ : def. $x^{i}$ ist Element von $x^{i+1}$. Jedes Element $x^{i}(i=1, \ldots, n-1)$ ist Mitglied in mindestens einem höheren Kontext $\mathrm{x}^{\mathrm{i}+1} \cdot \mathrm{x}^{\mathrm{i}+1}$ kann wiederum als Menge der es konstituierenden Einheiten der nächstniederen Ebene verstanden werden: $x^{i+1}=\left[x^{i} \mid x^{i} e x^{i+1}\right]$ $(i=1, \ldots, n-1)$. Man erhält dann $\epsilon$-Ketten der Form $x^{1} \in x^{2} \in x^{3}$ mit z.B. der folgenden möglichen Interpretation: der Schüler $x^{1}$ ist Mitglied in der Freundschaftsgruppe $x^{2}$ in der Schule $x^{3}$.

Um eine übersichtlichere Schreibweise zu erreichen, beschränken wir uns von nun an auf ein Zweiebenenmodell mit den Variablen $x, y, \ldots$ und $\mathrm{X}, \mathrm{Y}, \ldots$ für Individual- bzw. Gruppenebene. Dies ist keine Beschränkung der Allgemeinheit, da jedes Mehrebenenmodell formal immer als eine Kombination verschiedener, einander überlagernder Zweiebenenmodelle aufgefaßt werden kann. Im klassischen Zweiebenenmodell werden die Einheiten der Individualebene als äquivalent in Bezug auf die durch das Modell erfaßte strukturelle Differenzierung genau dann angesehen, falls sie zum gleichen Kontext gehören, formal:

$$
\mathrm{x} \text { äq. } \mathrm{y} \text { : def. ex. } \mathrm{X}(\mathrm{x} \in \mathrm{X} \text { und } \mathrm{y} \in \mathrm{X})(\mathrm{x}) \text {. }
$$

Der ,aggregative“ Ansatz des Modells wird deutlich, wenn man die Zuordnung eines Durchschnitts eines bestimmten Merkmals $m$ für den Kontext $\mathrm{X}$ auf seine Mitglieder $\mathrm{X}$ als ein Kontextmerkmal betrachtet:

$$
\mathrm{m}(\mathrm{x}, \mathrm{X}):=\sum_{\mathrm{y} \in \mathrm{X}} \mathrm{m}(\mathrm{y}) / \# \mathrm{X},
$$

wobei $\mathrm{m}(\mathrm{y})$ die Ausprägung des Merkmals $\mathrm{m}$ für $\mathrm{y}$ und \# $\mathrm{X}$ die Mächtigkeit von $\mathrm{X}$ bedeuten. $\bar{m}(\mathrm{x}, \mathrm{X})$ hängt nämlich überhaupt nicht von $\mathrm{x}$, sondern nur von $\mathrm{X} a b$, ist also von seiner Konstruktion her ein Gruppenmerkmal und wird hier als ein Kontextmerkmal im weiteren Sinne verwandt, da es allen Elementen $x, y$. . von $\mathrm{X}$ in gleicher Weise zugeordnet wird. Darin wird noch einmal der Sinn der Strukturhypothese (x) deutlich.

Struktur wird im klassischen Zweiebenenmodell allein durch die $\epsilon$-Relation dargestellt, die eine zweistellige Relation ist, die Elemente benachbarter Ebenen verbindet. Allgemein sollen Relationen, die Elemente verschiedener Ebenen verbinden, als Bestandteile der vertikalen Struktur angesehen werden. Neben der für das Modell grundlegenden $\epsilon$-Relation wären z.B. die Relationen , $\mathrm{X}$ ist zentral im Kontext $\mathrm{X}$ “ oder ,, $\mathrm{X}$ meidet den Kontext $X^{\text {" }}$ als Bestandteile der vertikalen Struktur anzusehen. Dagegen sollen Relationen, die Elemente der gleichen Ebene miteinander verbinden, als zur horizontalen Struktur gehörend verstanden werden. Solche Rela-

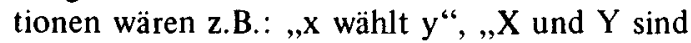
benachbart" oder , $\mathrm{x}$ und $\mathrm{y}$ sind durch einen Kommunikationskanal verbunden“. Die oben eingeführte Unterscheidung zwischen Kontextmerkmalen im engeren und weiteren Sinne Kontextmerkmale im engeren Sinne (KMieS) differenzieren zwischen den Elementen eines Kontextes, d.h. sie nehmen für verschiedene Elemente des gleichen Kontextes verschiedene Werte an, während Kontextmerkmale im weiteren Sinne allen Elementen eines Kontextes in gleicher Weise einen Wert zuordnen - kann nun formal wie folgt definiert werden:

$m(x, X)$ ist KMieS :def. ex. $x, y \in X m(x, X) \neq m(y, X)$.

Schematisch kann die Einteilung der Relationsund Funktionszeichen etwa folgendermaßen verdeutlicht werden:

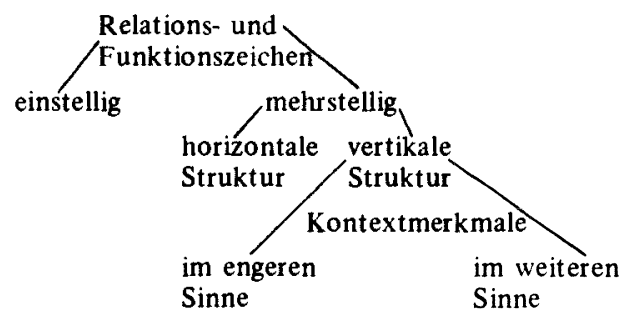




\section{Die Zerlegung relationaler Strukturen in Zonen relativer Verdichtung}

\section{a) Formulierung der Problemstellung in der Sprache der Graphentheorie}

Ausgangspunkt für die weiteren Überlegungen sei nun eine relationale Struktur oder - in der Sprache der Graphentheorie (Flament, 1963) ein gerichteter, markierter Graph (marked graph, Flament S. 46). Dazu gehören zunächst einmal eine (endliche) Menge von Punkten $S=[x, y, z$, ... ] und eine Menge von Linien zwischen den Punkten, d.h. eine Relation $R$ auf S ( $R$ ist eine Teilmenge des kartesischen Produktes von $\mathrm{S}$ mit sich selbst). In unserem Beispiel wäre $S$ die Menge der Schüler in den untersuchten Schulen und $R$ die Menge der Freundschaftswahlen der Schüler untereinander $-\mathrm{xRy}$ würde eine von $\mathrm{x}$ an y gerichtete Freundschaftswahl bedeuten. R ist also eine zweistellige, zur horizontalen Struktur der unteren Ebene gehörende Relation. Als Markierungen eines Graphen $\mathrm{G}=(\mathrm{S}, \mathrm{R})$ wollen wir in Erweiterung der bei Flament gegebenen Definition jede Funktion $\mathrm{m}$ auf $\mathrm{S}$ mit Werten in den reellen Zahlen ansehen, worunter dann insbesondere alle in einer Umfrage erhobenen Individualmerkmale fallen. Eine solche Funktion $\mathrm{m}$ wäre in unserem Beispiel unter anderem die Collegeabsicht, die für jeden Schüler $x$ aus $S$ je nachdem, ob er beabsichtigt, ein College zu besuchen oder nicht, die Werte $m(x)=1$ oder $\mathrm{m}(\mathrm{x})=\mathrm{O}$ annehmen würde. Es ist klar, daß auf diese Weise jedes erhobene Individualmerkmal der Schüler als Markierung $m$ des Graphen $\mathrm{G}=$ $(S, R)$ verstanden werden kann.

Im Sinne der oben angestellten Überlegungen zur Zerlegung relationaler Strukturen soll nun ein Verfahren angegeben werden, einen gegebenen Graphen $G=(S, R)$ in ,Zonen relativer Verdichtung “ (ZrV's) zu zerlegen ${ }^{3}$. Die dabei erhaltenen ZrV's sind dann die gesuchten primären Umwelten $X, Y, \ldots$ in dem vorgeschlagenen Dreiebenenmodell. Ausgehend von dem symmetrisierten Graphen $G_{S}:=(S, F)$, mit $\mathrm{xFy}$ :def. $\mathrm{xRy}$ und $\mathrm{yRx}$ - das Bestehen einer Freundschaftsbeziehung zwischen den Schülern

3 Für die Frage der Zerlegung in ZrV's haben eventuelle Markierungen des Graphen keine Bedeutung; sie spielen erst eine Rolle bei der Untersuchung der Homogenität von Merkmalsverteilungen in Bezug auf die relationale Struktur $\mathbf{R}$ (siehe 3.c). $\mathrm{x}$ und $\mathrm{y}$ wird genau dann angenommen, wenn sie sich gegenseitig als Freund angeben - werden auf Grund von noch genauer zu spezifizierenden Bedingungen Kanten aus $\mathrm{G}_{\mathrm{S}}$ entfernt und zwar mit der Absicht, auf diese Weise ein Zerfallen des Graphen in Zusammenhangskomponenten (Zshk) zu erreichen. Dabei verstehen wir unter Zshk (durch Kantenzüge) verbundene, maximale Teilgraphen von $\mathrm{G}_{\mathrm{S}}$ (siehe auch Flament S. 33). Je zwei Punkte einer Zshk müssen durch mindestens einen Kantenzug miteinander verbunden sein (Zusammenhang) und innerhalb von $\mathrm{G}_{\mathrm{S}}$ gibt es keinen weiteren Punkt, der zu der Zshk hinzugefügt werden könnte, ohne den Zusammenhang zu zerstören (Maximalität); eine Zshk ist also innerhalb des Gesamtgraphen isoliert ${ }^{4}$.

\section{Natürlich sind die so erhaltenen Zshk (ZrV's)} i.a. weder vollständig in Bezug auf die durch $F$ gegebene Struktur noch homogen in Bezug auf Markierungen des Graphen (relevante Individualmerkmale). Im Sinne der obigen Überlegungen ist daher eine Charakterisierung der Position der Schüler innerhalb der internen Struktur der Freunaschaftsgruppen durch Kontextmerkmale im engeren Sinne notwendig. Darüber hinaus müssen generelle Merkmale der Gruppenstruktur der Freundschaftsgruppen direkt als Gruppenmerkmale erfaßt werden; sie können dann auch als Kontextmerkmale im weiteren Sinne verstanden werden. Insgesamt ergibt sich dann ein Dreiebenenmodell bestehend aus Schülern, Zonen relativer Verdichtung der Freundschaftsnetzwerke und Schulen - Individualebene, primärer und sekundärer Umwelt entsprechend zusammen mit den entsprechenden strakturellen Charakterisierungen.

\section{b) Andere Verfahren zur Cliquenbestimmung}

Der im folgenden darzustellende Zerlegungsalgorithmus erhebt den Anspruch, insbesondere bei großen Freundschaftsnetzwerken eine für eine weitere statistische Analyse im Rahmen des oben angegebenen Dreiebenenmodells angemessene Zerlegung in primäre Umwelten zu leisten. Es erscheint daher sinnvoll, kurz auf andere vorhandene Ansätze zur ,Cliquenbe-

4 Zshk wären z.B. isolierte Punkte oder isolierte Paare oder jede andere in sich verbundene Teilstruktur des Graphen, die durch keine Kante mit dem Rest des Graphen verbunden ist. 
stimmung" einzugehen und sie auf ihre Anwendbarkeit für unsere Problemstellung zu überprüfen. Die vorgeschlagenen Methoden lassen sich einteilen in

1. Methoden, die durch Umordnen der Zeilen und Spalten der Soziomatrix eine Häufung der Wahlen um die Hauptdiagonale erreichen (z.B. Forsyth u. Katz, 1946; Beum u. Brundage, 1950; Coleman u. McRae, 1960)

2. Methoden, die auf der Potenzierung der Soziomatrix beruhen (z.B. Festinger, 1949; Luce u. Perry, 1949; Luce, 1950; Harary u. Ross, 1957)

3. Methoden, die die Dimensionalität soziometrischer Daten durch Faktorenanalyse oder multidimensionale Skalierung reduzieren (z.B. McRae 1960; Gleason, 1967; Langeheine, 1976)

4. Clusteranalytische Verfahren (z.B. Bock u. Husain, 1950; Peay, 1975).

Läßt man die Schwierigkeiten, die sich bei der praktischen Umsetzung der Verfahren 2, - 4 . bei umfangreichen Netzwerken wegen der begrenzten Speicher- und Rechenkapazitäten der verfügbaren Rechenanlagen ergeben, einmal außer Betracht, so kann man in Hinblick auf die Verwendbarkeit dieser Verfahren für unsere Problemstellung folgendes feststellen:

Die unter 1. und 3. angegebenen Verfahren sind keine eigentlichen Verfahren zur Cliquenbestim. mung; sie dienen eher zur Aufbereitung des Datenmaterials und erheben den Anspruch, auf diese Weise die Cliquenbestimmung $\mathrm{zu}$ erleichtern. So werden bei den Umordnungsalgorithmen Mitglieder einer Clique in den meisten Fällen näher (im Sinne der Nachbarschaft in der Soziomatrix) aneinanderrücken, jedoch bleibt die Frage nach Kriterien zur Abgrenzung der Cliquen untereinander unbeantwortet. Gleiches gilt für die faktorenanalytischen Verfahren, wo aus dem Vorkommen ,hoher Faktorladungen“ auf die Zugehörigkeit zu gewissen Untergruppierungen geschlossen wird - analoge Schwierigkeiten ergeben sich bei den Verfahren der multidimensionalen Skalierung. Außerdem weisen Ähnlichkeiten in den Faktorladungen nicht unbedingt auf eine Zugehörigkeit zur gleichen Clique, sondern lediglich auf eine Ähnlichkeit der Beziehungsmuster zu anderen Mitgliedern des Netzwerkes hin - eine notwendige, aber nicht hinreichende Bedingung für die Zugehörigkeit zur gleichen Clique. Generell ist die Wahl eines geeigneten
Nähemaßes bei den unter 3. angeführten Verfahren problematisch, eine Tatsache, die aber auch wegen der durch die Vielzahl der vorgeschlagenen Nähemaße erreichbaren Flexibilität der Verfahren als Vorteil angesehen werden kann. Wesentlicher Nachteil der unter 1. und 3. angegebenen Verfahren bleibt aber das Fehlen einer expliziten Cliquendefinition, ohne die natürlich kein Verfahren zur Cliquenbestimmung angegeben werden kann. Die Entscheidung, nach welchen Kriterien die Cliquen voneinander abzugrenzen sind, muß nach Anwendung der Verfahren ad hoc getroffen werden.

Dagegen werden bei den unter 2. und 4. angegebenen Verfahren mehr oder weniger explizite Cliquendefinitionen vorgegeben. So definieren Luce und Perry eine Clique als maximalen vollständigen Teilgraphen und Luce eine n-Clique als maximalen Teilgraphen derart, daß je zwei seiner Punkte durch einen Weg der Länge kleiner gleich $n$ miteinander verbunden sind. Die erste Definition ist sehr restriktiv und führt, wie Harary und Ross zeigen, zu einer Zerlegung von Gruppierungen mit hoher Interaktionsdichte in eine Vielzahl sich überlappender Cliquen. Ein weiterer Nachteil ist, daß zentralisierte, nicht hochintegrierte Strukturen nicht entdeckt werden können, da sie nicht unter die Cliquendefinition fallen. Die verallgemeinerte Definition der n-Clique dagegen läßt auch Wege zu, die nicht innerhalb der zu bestimmenden Clique verlaufen müssen. d.h. die Mitgliedschaft in der Clique kann eventuell durch Kanten, die aus der Clique heraus fuhren, erreicht werden. Besonders störend wirken sich bei dieser Definition Überlappungen aus.

Auch die von Peay vorgeschlagene nichtmetrische hierarchische Zerlegungsprozedur orientiert sich an der Definition der n-Clique von Luce. Als Ausgangspunkt der Prozedur wird ein bewerteter, vollständiger und symmetrischer Graph genommen, wobei die Bewertungen der Linien des Graphen jeweils als Distanzen zwischen den entsprechenden Punkten gedeutet werden. Je nach der Festlegung von Schwellenwerten, bis $z u$ denen Distanzen zwischen den Punkten berücksichtigt werden sollen, ergibt sich dann eine in sich aufsteigend geordnete Folge von Zerlegungen in Cliquen. Der Vorteil dieser Zerlegungsprozedur ist es, daß durch die Konstruktion einer Hierarchie von Zerlegungen verfahrenstechnisch im Vergleich zur Methode von 
Harary und Ross einige Vereinfachungen und Verallgemeinerungen gelingen. In Hinblick auf die Verwendbarkeit des Verfahrens zur Bestimmung von primären Umwelten gelten die gleichen Einwände wie oben. Zusätzlich bleibt die geeignete Wahl eines Schwellenwertes, der die endgültige Zerlegung bestimmen soll, problematisch.

Eine mehr implizite Cliquendefinition liegt dem Verfahren von Bock und Husain zugrunde. Auf der Basis eines Distanzmaßes - in unserem Falle etwa der graphentheoretischen Distanz (siehe 3.a) - zwischen den einzelnen Punkten des Netzwerkes wird der Prozeß des Hinzufügens neuer Punkte zu bereits bestehenden Clustern durch den B-Koeffizienten von Holzinger gesteuert. Er wird so lange fortgesetzt, ,until a sharp drop appears in the value of B" (S. 149). Da keine weiteren Angaben gemacht werden, was genau unter einem signifikanten Absinken von B zu verstehen ist, bleibt auch bei diesem Verfahren das kritische Problem der Abgrenzung der Cliquen untereinander ungelöst.

\section{c) Ein neuer Vorschlag zur Zerlegung von Netz- werken}

Das Verfahren von Bock und Husain gibt aber gerade durch seinen Verzicht auf eine explizite Cliquendefinition Hinweise auf andere Möglichkeiten, an das Problem der Cliquenbestimmung heranzugehen. Ausgangspunkt ist die Vorstellung, daß eine Clique nicht so sehr durch ihre interne Struktur (z.B. maximaler vollständiger Teilgraph), sondern eher in Bezug auf ihre Begrenzung zum übrigen Netzwerk definiert wird. Man sollte daher besser von einer Zerlegung in „Zonen relativer Verdichtung“ sprechen eine solche $\mathrm{ZrV}$ ist dann nicht notwendig eine hochintegrierte Struktur; sie ist eine Einheit nur in dem Sinne, daß sie im Vergleich zu ihrer Umgebung verdichtet ist. Durch einen Verzicht auf eine explizite Cliquendefinition wird vor allem die Selektivität der Verfahren überwunden, die auf dem Absuchen von soziometrischen Netzwerken nach bestimmten, in der Cliquendefinition vorgegebenen Katalogen von Interaktionsmustern beruhen, und damit das Auffinden anderer, in der Cliquendefinition nicht erfaßter Interaktionsmuster unmöglich macht.

Gesucht werden müssen also Kriterien, die es erlauben, mit Hilfe eines Algorithmus solche
„Bruchstellen“ $z u$ finden, die eine $\mathrm{ZrV}$ vom übrigen Netzwerk trennen. Ziel des Verfahrens ist es dann, das Gesamtnetzwerk an Hand dieser Bruchstellen in ,Scherben“ zu zerschlagen. Dadurch wird eine Analyse des gesamten Netzwerkes möglich, die die Bestimmung und Analyse von Cliquen im Sinne der eingeschränkten Definition von Luce und Perry als Spezialfall enthält. Dabei verlagert sich der methodische Ansatz von der Clusteranalyse weg zu graphentheoretischen Fragestellungen wie der Bestimmung von Schnittpunkten und Brïcken ${ }^{5}$. Brïcken (bridges) und Schnittpunkte (cut points) sind nämlich in der Sprache der Graphentheorie gerade Bezeichnungen für Bruchstellen im oben gemeinten Sinne (Harary, Norman u. Cartwright, 1965 , Kap. 7 u. 8, insbesondere S. 198 ff und S. $228 \mathrm{ff}$ ). In unserem Zusammenhang können Brücken definiert werden als Kanten, deren Entfernung einen sonst zusammenhängenden Graphen in zwei Zusammenhangskomponenten zerfallen läßt. Entsprechend sind Schnittpunkte Punkte eines Graphen, deren Entfernung ein Zerfallen des Graphen in verschiedene Zshk zur Folge hat.

\section{d) Darstellung des Zerlegungsalgorithmus ${ }^{6}$}

Der folgende Zerlegungsalgorithmus orientiert sich nicht an einer a priori vorgebenen Cliquen-

5 Eine solche Vorgehensweise entspricht der von Weiss und Jacobson (1955) angewandten Methode zur Analyse der Interaktionsstruktur innerhalb einer Organisation. Weiss und Jacobson beschreiben ihren Ansatz wie folgt: ,It seemed on theoretical, intuitive, and practical grounds that groups' of individuals should be defined in terms of their degree of inner connectedness, if the ultimate objective is the description of a complex organization consisting of a set of groups" (S. 662). „The basic approach to the determination of the structure of the organizations was to isolate the separate work groups by removing liaison persons and omitting contacts between groups" (S. 664). Die Durchführung des Verfahrens mit einer $196 \times 196$ Matrix lieferte 22 Arbeitsgruppen, auf die insgesamt $80 \%$ der Interaktionen entfielen. Weiss und Jacobson geben jedoch keinen Algorithmus zur Bestimmung der Arbeitsgruppen an - die Zerlegung der Ausgangsmatrix in sogenannte Segmente und die endgültige Identifikation der Arbeitsgruppen geschah per Inspektion.

6 Für die einzelnen Schritte des Zerlegungsalgorithmus wurden Computerprogramme entwickelt (siehe Kappelhoff, 1976). Leider ist das Programmpaket insgesamt - insbesondere was die Eingabeformate angeht - nicht sehr flexibel, so daß bei einer eventuellen Anwendung auf andere Daten eine Adaption erforderlich wäre. 
definition, sondern erreicht das Zerfallen des Ausgangsnetzwerkes (eines symmetrischen Graphen) in immer kleinere Zshk durch Aufsuchen von Brücken und Schnittpunkten und Entfernen der entsprechenden Kanten (Schnittprozeduren), jeweils gefolgt von den dadurch möglich gewordenen Zerlegungen in Zshk. Ein Vorteil des Verfahrens ist es insbesondere, daß zur Bestimmung der Schnitte nur Informationen über die nähere Umgebung der Schnittstelle benötigt werden, d.h. Punkte, die über einen Weg bis zur Länge 3 erreicht werden können. Es werden also nur Brücken und Schnittpunkte im lokalen Sinne gesucht; entsprechend ist das infolge der Schnittprozeduren mögliche Zerfallen in Zshk auch nur im lokalen Sinne gesichert.

Einen Weg der Länge $n$ mit den Elementen $x_{0}$, $\mathrm{x}_{1}, \ldots, \mathrm{x}_{\mathrm{n}}$ wird Kette der Länge $\mathrm{n}$ genannt, falls die Endpunkte $x_{0}$ und $x_{n}$ jeweils mindestens 3 und die $Z$ wischenglieder $x_{1}, \ldots, x_{n}-1$ alle genau 2 erwiderte Wahlen haben. Für das Legen von Schnitten sollen die folgenden drei Gesichtspunkte maßgebend sein:

1. Es sollen keine Zyklen (geschlossene Wege) der Länge 3 und 4 (Dreiecke und Vierecke) geschnitten werden, da sie als Kerngruppen bzw. als Kondensationspunkte von Gruppen angesehen werden; dagegen können Zyklen der Länge 5 in ungünstigen Fällen geschnitten werden.

2. Diese Bestimmung kann nur unscharf formuliert werden; sie wird durch die Schnittalgorithmen konkretisiert. Ketten sollen nur dann geschnitten werden, wenn sich an beiden Enden der Kette „Verdichtungen“ befinden, die dann als Kerngruppen interpretiert werden können.

3. Haben zwei Gruppen einen Punkt gemeinsam und besteht sonst keine Verbindung zwischen den Elementen der beiden Gruppen (Liaisonperson), so soll ebenfalls ein Schnitt gelegt werden.

Der Zerlegungsalgorithmus zerfällt in die folgenden Schritte:

1. Zerlegung des Ausgangsnetzwerkes in Zshk.

2. Schnittprozedur für Ketten der Länge größer gleich 2.

Die Prozedur sucht das Netzwerk auf Ketten der Länge größer gleich 2 ab und legt bei Vorliegen der unten genauer angegebenen Schnittbedingungen die entsprechenden Schnitte.
Ketten der Länge 3 werden genau dann geschnitten, wenn es zwischen ihren Endpunkten keine Kanten und keinen Weg der Länge 2 gibt; es können dann insbesondere keine Dreiecke oder Vierecke geschnitten werden. Ketten mit einer Länge größer als 3 werden in jedem Fall geschnitten, da nicht befürchtet werden muß, Dreiecke oder Vierecke zu zerschneiden. In beiden Fällen wird die Tatsache, daß die Endpunkte einer Kette nach Definition mindestens 3 erwiderte Wahlen aufweisen müssen, als ausreichender Anhaltspunkt für das Vorliegen von Verdichtungen an den Kettenenden angesehen. Es ist klar, daß der Begriff ,Verdichtung" in diesem Zusammenhang nur relativ zu dem wenig integrierten Netzwerkbereich gesehen werden kann, in dem der Schnitt gelegt werden soll. Dabei wird das Vorliegen eines lokal nur schwach integrierten Netzwerkbereiches durch das Vorhandensein langer Ketten indiziert.

Analog verläuft die Schnittprozedur für Ketten der Länge 2. Wegen der kleineren Kettenlänge werden aber zusätzliche Bedingungen eingeführt, die prüfen, ob die Endpunkte der Kette zu verschiedenen Gruppierungen gehören, d.h. nicht in dem oben definierten lokalen Sinne - außer durch die betrachtete Kette - miteinander verbunden sind. Außerdem werden zusätzlich geeignete Bedingungen für das Vorliegen von Verdichtungen an den beiden Kettenenden definiert ${ }^{7}$.

Diese Schnittprozedur sucht also alle Ketten der Länge 2 und größer auf, prüft sie auf lokales Zerfallen in zwei Gruppierungen und auf das Vorliegen von Verdichtungen an den Kettenenden und schneidet bei Erfüllung der geforderten Kriterien eine geeignete Kante innerhalb der Kette.

3. Erneutes Zerlegen in Zshk.

4. Schnittprogramm für Ketten der Länge 1.

Die erforderlichen Bedingungen für das Schneiden von Ketten der Länge 1 werden gemäß den oben aufgestellten Kriterien 1 und 2 in Analogie zu denen für Ketten der Länge 2 formuliert ${ }^{7}$.

7 Das Vorliegen kürzerer Ketten wird allgemein als Indiz für dichtere Netzwerkstrukturen angesehen, so daß schärfere Kriterien für das Vorliegen von Verdichtungen an den Kettenenden aufgestellt werden. Dies hat zur Folge, daß sich das Verfahren in Abhängigkeit von der lokalen Netzwerkdichte selbst steuert. Zur genaueren Darstellung der aufgestellten Bedingungen siehe Kappelhoff (1976). 
5. Erneutes Zerlegen in Zshk.

6. Schnittprozedur für Liaisonpersonen.

Unter einer Liaisonperson soll eine Person verstanden werden, die gleichzeitig zwei sonst unverbundenen Gruppen angehört. Offenbar können solche Gruppen nur durch das Herausnehmen der Liaisonperson aus dem Netzwerk getrennt werden ${ }^{8}$. Dies geschieht durch die nun zu erläuternde Schnittprozedur.

Zunächst wird das Netzwerk auf mögliche Kandidaten $z$ für eine Liaisonperson abgesucht. Dazu muß $\mathrm{z}$ mindestens 4 erwiderte Wahlen haben, denn jede der beiden noch zu bildenden Komponenten soll mindestens 2 Personen umfassen; außerdem müssen die Gewählten selbst jeweils mindestens 2 erwiderte Wahlen haben. Ist ein solches $\mathrm{z}$ gefunden, wird die Menge $\mathrm{W}_{\mathrm{Z}}$ der von $z$ Gewählten auf folgende Art in Komponenten zerlegt: die schon vorhandenen Kanten und die Wege der Länge 2 - die auch über nicht $\mathrm{zu} \mathrm{W}_{Z}$ gehörende Punkte mit Ausnahme von z selber führen können $-z$ wischen den Punkten von $W_{Z}$ werden als Kantenmenge eines neuen Graphen mit der Punktmenge $W_{Z}$ angesehen. Die Komponenten von $z$ werden dann gerade als Zshk dieses neuen Graphen definiert. Auf diese Weise wird erreicht, daß jeder Punkt einer Komponente mindestens über einen Weg der Länge 2 der dann nicht über $z$ läuft - mit einem anderen Punkt der Komponente verbunden ist. Punkte aus verschiedenen Komponenten sind dagegen nicht - auch nicht durch indirekte, nicht über $\mathrm{z}$ laufende Wahlen - miteinander verbunden. Damit wird gesichert, daß eventuell vorhandene Gruppen, die die Komponenten von $z$ als Kerngruppen enthalten, nur durch $\mathrm{z}$ miteinander verbunden sind; $z$ ist also lokaler Schnittpunkt im oben definierten Sinne, d.h. nach Entfernen von $\mathrm{z}$ zerfällt der Graph lokal. Falls z Liaisonperson sein soll, muß $W_{Z}$ in genau 2 Kerngruppen (Komponenten) mit mindestens der Mächtigkeit 2 zerfallen. Um die Interpretation als Kerngruppen für die Komponenten von $W_{Z}$ zu rechtfertigen, wird zusätzlich verlangt, daß jede Kompo-

8 Im weiteren Verlauf der Analyse wird die Liaisonperson vorübergehend beiden Gruppen zugeordnet, wobei jeweils die Freundschaftsbeziehungen zu den Mitgliedern der anderen Gruppe geschnitten werden. Die endgültige Zuordnung zu einer der beiden Gruppen geschieht dann auf der Grundlage der relativen Zentralitäten in den beiden Gruppen (siehe 3.b). nente entweder 3 Punkte enthält oder daß alle Punkte der Komponente mindestens eine erwiderte Wahl haben, die nicht an ein anderes Mitglied der Komponente oder an $\mathrm{z}$ geht. Sind alle diese Bedingungen erfüllt, wird $\mathrm{z}$ als Liaisonperson angesehen und ein Schnitt gelegt.

\section{Erneutes Zerlegen in Zshk.}

Als Resultat ergeben sich die gesuchten Zonen relativer Verdichtung. Eine solche $\mathrm{ZrV}$ kann also definiert werden als eine Zshk des durch die angegebenen Schnittprozeduren zerlegten Ausgangsnetzwerkes ${ }^{9}$.

Das oben dargestellte Verfahren wurde auf die „Adolescent-Society“"-Daten von Coleman (1961) angewandt, die unter anderem eine Totalerhebung der Freundschaftsnetzwerke an den untersuchten High Schools enthalten (siehe Kappelhoff, 1976). Dabei ergab sich:

1. Dort, wo hochintegrierte Netzwerkbereiche vorherrschen, gelingt eine befriedigende Zer. legung in ZrV's, und zwar in der Regel durch Trennung von Gruppierungen, die durch Liaisonpersonen oder Ketten der Länge 1 verbunden sind. Die entstandenen ZrV's haben durchweg Dreiecke und Vierecke als Kerne und weisen eine hohe Integration und eine geringe Zentralisierung auf.

2. In wenig integrierten Netzwerkbereichen erscheint die Zerlegung in ZrV's nicht völlig befriedigend und oft von einer gewissen Willkür bestimmt. Die entstandenen ZrV's haben meistens einen zentralen Schüler und mehrere davon ausgehende Ketten. Die Integration ist dementsprechend gering und die Zentralisierung hoch.

Sicher wird die weitere praktische Arbeit mit dem Zerlegungsalgorithmus dazu Anlaß geben, manche der angegebenen Schnittbedingungen zu überprüfen und zu modifizieren; auch sind andere Kriterien zur Steuerung der Schnittprozeduren denkbar. Unabhängig davon bleibt eine grundsätzliche Schwierigkeit: je homogener die Ausgangsstruktur ist, d.h. je weniger man von dem Vorhandensein ausgeprägter Gruppierungen im Sinne von Netzwerkverdichtungen sprechen

9 Durch die Schnittprogramme werden zusätzlich noch Punkte bestimmt, die sich durch eine besondere Stellung im Netzwerk auszeichnen, so z.B.: Punkte in Ketten, Punkte an Schnittstellen von Ketten und Brücken und Liaisonpersonen. 
kann, um so weniger sinnvoll wird die Anwendung eines solchen Zerlegungsalgorithmus, da das Steuerungsprinzip der Schnittprozeduren auf der Suche von Verbindungen zwischen Verdichtungen beruht. Ziel des Verfahrens kann also keine in allen denkbaren Einzelfällen zufriedenstellende Gruppenbestimmung sein. Vielmehr wird eine Zerlegung von empirisch ermittelten Netzwerken großen Umfangs in primäre Umwelten (ZrV's) erreicht, die dann als Grundlage einer Netzwerkanalyse mit statistischen Methoden dienen kann.

\section{Die Charakterisierung von soziometrischen Strukturen auf der Grundlage einer Metrik}

Da sich die vorgeschlagene Zerlegung relationaler Strukturen in primäre Umwelten nicht an den Prinzipien maximaler Vollständigkeit und Homogenität orientiert, ergibt sich im Sinne der in 1.c angestellten Überlegungen die Notwendigkeit, für eine eingehendere Strukturanalyse die Positionen der Elemente der ZrV's in Hinblick auf deren interne Struktur (durch Strukturindizes) sowie in Hinblick auf die Verteilung relevanter Individualmerkmale (durch Homogenitätsindizes) genauer zu charakterisieren. Dies geschieht auf der Grundlage einer Metrisierung der internen Strukturen der ZrV's. Welche der vielen denkbaren positionalen Charakterisierungen sich fur die je konkrete Untersuchung als sinnvoll erweisen, kann naturlich nicht allgemein beantwortet werden. Hier sollen lediglich noch einige Struktur- und Homogenitätsindizes angegeben werden, die von allgemeinem Interesse sein dürten, da sie im Sinne des angegebenen Dreiebenenmodells eine sinnvolle Ergänzung zum vorgeschlagenen Zerlegungsalgorithmus darstellen ${ }^{10}$.

\section{a) Metrisienung der internen Strukturen der ZrV's auf der Grundlage der graphentheore- tischen Distanz}

Die Distanz d (x, y) zwischen den Punkten $x$ und $y$ einer $\mathrm{ZrV} Z$ wird als eine modifizierte

\footnotetext{
10 Auch für die Metrisierungsprozedur sowie die Berechnung der im folgenden angegebenen Strukturund Homogenitätsindizes wurden Computerprogramme entwickelt (siehe Kappelhoff, 1976).
}

graphentheoretische Distanz bestimmt. Die graphentheoretische Distanz zwischen zwei Punkten eines verbundenen symmetrischen Graphen - die ZrV's können nach Konstruktion immer als verbundene symmetrische Teilgraphen des Ausgangsgraphen $G_{S}=(S, F)$ interpretiert werden - ist definiert als Länge des kürzesten Weges zwischen $\mathrm{x}$ und $\mathrm{y}, \mathrm{d} . \mathrm{h}$. für direkt durch eine Kante verbundene Punkte ergibt sich eine Distanz von 1 und für nur indirekt durch einen Kantenzug der Länge 2 verbundene Punkte eine Distanz von 2 usw. (siehe Flament, 1963:25). Diese Definition wird nun erweitert, indem neben einfachen Kanten auch andere Strukturelemente zum Aufbau von Wegen zugelassen werden, nämlich Dreiecke und Vierecke. Durch geeignete Wichtung der Strukturelemente - etwa Dreiecke mit 0,75 und Vierecke mit 1,5; Kanten werden weiterhin mit 1 bewertet kann auch den so gebildeten Wegen in eindeutiger Weise eine Länge zugeordnet werden. Die so modifizierte Distanzdefinition erfült (ebenso wie die graphentheoretische Distanz) die Eigenschaften einer Metrik im strengen mathematischen Sinne (siehe Kappelhoff, 1976). Der Sinn dieser verallgemeinerten Definition liegt in der Möglichkeit, bestimmte Konstellationen, denen vielleicht vom theoretischen Ansatz her das besondere Interesse gilt, besonders wichten $\mathrm{zu}$ können. So wurden in unserem Falle Dreiecke und Vierecke als weitere Strukturelemente gewählt, weil sie als Kristallisationspunkte von Gruppen angesehen werden. Durch entsprechende Setzung der zugehörigen Gewichte wird eine relative Schrumpfung der Distanzen in integrierten Gruppen und damit eine angemessenere Widerspiegelung der Gruppenstruktur in der Distanzmatrix erreicht.

\section{b) Die interne Struktur der ZrV's}

Für die Analyse der relationalen Struktur der ZrV's werden die in den zugeordneten Distanzmatrizen vorhandenen Informationen in Indizes zusammengefaßt. Die folgende Darstellung der strukturellen Parameter Zn, I und HZ basiert auf einer Arbeit von Hфivik und Gleditsch (1969).

Ein Mitglied einer $\mathrm{ZrV}$ ist um so zentraler, je geringer sein Abstand von den anderen Mitgliedern der $\mathrm{ZrV}$ ist, formal:

$$
\left.\operatorname{Zn}(\mathrm{x}, \mathrm{Z}):=\sum_{\mathrm{y} \in \mathrm{Z} d} \mathrm{~d}, \mathrm{y}\right) \text {. }
$$


$\mathrm{Zn}$ charakterisiert die Position von $\mathrm{x}$ in $\mathrm{Z}$, ist also ein Kontextmerkmal im engeren Sinne. Ein $\mathrm{Maß}$ für die Gruppenintegration bzw. -kohäsion erhält man durch die Aggregation von $\mathrm{Zn}$ über alle Mitglisder $x$ der $\mathrm{ZrVZ}$, formal:

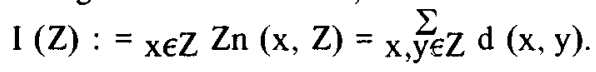

I beschreibt die $\mathrm{ZrV}$ als Ganzes, ist also ein Gruppenmerkmal und kann auch als Kontextmerkmal im weiteren Sinne verstanden werden. Die Verteilung der Zentralitäten innerhalb einer $\mathrm{ZrV}$ gibt Aufschluß über den Grad der Zentralisierung der $\mathrm{ZrV}$; sie heißst zentralisiert, falls große Unterschiede zwischen den Zentralitäten ihrer Elemente bestehen. $\mathrm{HZ}$ wird wie folgt definiert:

$$
\mathrm{HZ}(\mathrm{Z}):=\sum_{\mathrm{x} \in \mathrm{Z}}(\mathrm{Zn}(\mathrm{x}, \mathrm{Z})-\min [\mathrm{Zn}(\mathrm{y}, \mathrm{Z}) \mid \mathrm{y} \in \mathrm{Z}])
$$

$\mathrm{HZ}$ mißt also die Homogenität in den Positionen der Elemente einer $\mathrm{ZrV}$ als Abweichung von dem minimalen $\mathrm{Zn}$-Wert. Durch lineare Normierung werden die Indizes jeweils für verschiedene Gruppengrößen vergleichbar gemacht.

\section{c) Die Homogenität von Markierungen (Merk- malen) in Bezug auf die metrische Struktur der ZrV's}

Unter einer Markierung einer Struktur verstehen wir eine Zuordnungsvorschrift $\mathrm{m}$, die auf der Menge der die Struktur konstituierenden Einheiten definiert ist und jeder Einheit eine reelle Zahl (Merkmalsausprägung) zuordnet (siehe 2.a). Es soll nun die Homogenität solcher Merkmalsverteilungen in Bezug auf die in der Distanzmatrix D dargestellte metrische Struktur einer $\mathrm{ZrV}$ $\mathrm{Z}$ untersucht werden ${ }^{11}$. Dies bedeutet, daß man

11 Ein Ähnlichkeitsmaß für nominale Merkmale, das auf Freundschaftspaaren als Analyseeinheit basiert, wurde von Coleman (1958, Appendix B und 1966, Appendix E) entwickelt. Die Freundschaftswahlen werden nach den Eigenschaften der Wähler und Gewählten kreuztabelliert und dann die tatsächlichen mit den auf Grund der Randverteilungen bei zufälliger Assoziation zu erwartenden Zellbesetzungszahlen verglichen. Als Ähnlichkeitsmaß wird dann der Grad genommen, zu dem der Erwartungswert in Bezug auf den maximal möglichen Wert überschritten wird. Die Berücksichtigung der Erwartungswerte macht das $\mathrm{Ma} ß$ unabhängig von der Randverteilung des betrachteten Merkmals in der zugrunde liegenden Gruppe; gemessen wird also die Ähnlichkeit von Freundschaftspaaren im Rahmen einer Relationalanalyse unabhängig von der sozialen Zusammensetzung des Relationsgefüges. Die Begrenzung des dabei zwei Aspekte eines Sozialsystems miteinander verbindet, nämlich die im Mehrebenenmodell erfaßte relationale Zuordnung der Einheiten als unterliegende Struktur und eine Markierung dieser Struktur durch Individualmerkmale. Position und Eigenschaften des Positionsinhabers werden so zueinander in Beziehung gesetzt.

Ausgangspunkt zur Bestimmung der Homogenitätsposition eines Elements $\mathrm{x} \% \mathrm{O} \mathrm{Z}$ ist also einmal seine Position innerhalb von $Z$ und zum anderen die darauf bezogene Verteilung des betrachteten Merkmals m. Auftretende Inhomogenitäten $m(x)-m(y)$ zwischen den Netzwerkmitgliedern $x$ und $y$ werden je nach deren Nähe innerhalb von $Z$ verschieden gewichtet, so daß Inhomogenitäten $z w i s c h e n$ benachbarten Elementen stärker berücksichtigt werden als solche zwischen weiter entfernten. Die Wichtungsfaktoren $w_{x y}$ werden dabei als inverse Distanzen aus $D$ übernommen $\left(w_{x y}:=1 / d(x, y)\right.$. Formal ergibt sich die Homogenitätsposition von $\mathrm{x}$ in $\mathrm{Z}$ wie folgt:

$$
\begin{aligned}
\operatorname{HP}^{2}(\mathrm{x}, \mathrm{Z}):= & \sum_{\substack{\mathrm{y} \in \mathrm{Z} \\
\mathrm{y} \neq \mathrm{x}}}(\mathrm{m}(\mathrm{x})-\mathrm{m}(\mathrm{y}))^{2} \cdot \mathrm{w}_{\mathrm{xy}} / \\
& \sum_{\substack{\mathrm{y} \in \mathrm{Z} \\
\mathrm{y} \neq \mathrm{x}}} w_{\mathrm{xy}} \cdot{ }^{12}
\end{aligned}
$$

$\operatorname{HP}^{2}(\mathrm{x}, \mathrm{Z})$ ist also gerade die durch die Nähen $w_{x y}$ gewichtete Summe der quadrierten Inhomogenitäten zwischen $x$ und allen anderen Elementen $y$ aus $Z$. Die Summe der Nähen $w_{x y}$ über alle Elemente $y$ aus $Z$ (mit der Ausnahme von $x$ selbst) kann als ein Maß für die Zentralität von $x$ in $Z$ aufgefaßt werden, das auf Nähen an der Stelle von Distanzen basiert. Daraus ergibt sich, daß die Homogenitätsposition eines Elementes $\mathrm{x}$ in $\mathrm{Z}$ unabhängig ist von der Zentralität dieses Elements. HP beschreibt die Homogenitätsposition von $\mathrm{x}$ in $\mathrm{Z}$ und ist daher ein Kontextmerkmal im engeren Sinne. Mit Hilfe von HP ist eine Analyse von Entmischungsvorgängen in relationalen Strukturen möglich, so z.B. die Untersuchung der Frage, inwisweit Freundschaftsnetz-

Maßes liegt darin, daß nur die totale Netzwerkhomogenität als Gruppenmerkmal gemessen werden kann, nicht aber die Homogenitätsposition der Gruppenmitglieder als Kontextmerkmal im engeren Sinne.

12 Offenbar ist das vorgeschlagene Homogenitätsmaß nur sinnvoll für Dichotomien oder mindestens intervallskalierte Merkmale, da nur in diesen Fällen die Differenz $m(x)-m(y)$ sinnvoll als Inhomogenität interpretiert werden kann. 
werke von Schülern homogen sind in Bezug auf interessierende Merkmale wie sozialökonomischer Status der Eltern, Freizeitaktivitäten, Status im informellen System der Schulen usw. (Kappelhoff, 1976).

\section{d) Zusammenfassende Darstellung des Dreiebe- nenmodells}

Bei Verwendung der eingeführten Systematik läßt sich das betrachtete Dreiebenenmodell wie folgt formal darstellen:

\section{Ebenen des Modells (siehe 1.c):}

$\mathrm{x}^{1}, \mathrm{y}^{1}, \ldots$ : Individualebene (Schüler)

$\mathrm{x}^{2}, \mathrm{y}^{2}, \ldots$ : Ebene der primären Umwelten (ZrV's, die durch die Zerlegung der Freundschaftsnetzwerke an den einzelnen Schulen gewonnen werden ( siehe 2.d))

$x^{3}, y^{3}, \ldots$ : Ebene der sekundären Umwelten (Schulen)

\section{Metrisierung der Strukturen der ZrV's (siehe} 3. $a)$ :

$d\left(x^{1}, y^{1}\right)$ : aus der internen Struktur der ZrV's abgeleitete Distanzen zwischen den Schülern. $d$ ist ein zur horizontalen Struktur gehöriges zweistelliges Funktionszeichen.

\section{Kennzeichnung der internen Struktur der ZrV's (siehe 3.b):}

$\operatorname{Zn}\left(\mathrm{x}^{1}, \mathrm{x}^{2}\right)$ : Zentralität des Schülers $\mathrm{x}^{1}$ in der $\mathrm{ZrV}$ $\mathrm{x}^{2} ; \mathrm{Zn}$ ist ein zur vertikalen Struktur gehöriges zweistelliges Funktionszeichen (Kontextmerkmal im engeren Sinne).

$\mathrm{I}\left(\mathrm{x}^{2}\right)$ : Integration der $\mathrm{ZrV} \mathrm{x}^{2} ; \mathrm{I}$ ist Gruppenmerkmal und kann gemäß der Strukturhypothese $(\mathrm{x})$ auch als Kontextmerkmal im weiteren Sinne verstanden werden.

$\mathrm{HZ}\left(\mathrm{x}^{2}\right)$ : Zentralisierung der $\mathrm{ZrV} \mathrm{x}^{2}$. Der formale Status von $\mathrm{HZ}$ ist der gleiche wie von $\mathbf{I}$.

\section{Homogenität von Merkmalsverteilungen / siehe 3.c):}

HP $\left(x^{1}, x^{2}\right)$ : Homogenitätsposition des Schülers $\mathrm{x}^{1}$ in der $\mathrm{ZrV} \mathrm{x^{2 }}$. Der formale Status von $\mathrm{HP}$ ist der gleiche wie von $\mathrm{Zn}$.

\section{Literatur:}

Beum, C.O., und Brundage, E.G., 1950: A Method for Analyzing the Sociomatrix. Sociometry 13.

Bock, R.D., und Husain, S.Z., 1950: An Adaption of Holzingers B-Coefficients for the Analysis of Sociometric Data. Sociometry 13.

Campbell, E.Q., und Alexander, C.N., 1965: Structural Effects and Interpersonal Relationships. American Journal of Sociology 71.

Coleman, J.S., 1958: Relational Analysis. Human Organization 17.

Coleman, J.S., 1961: The Adolescent Society. New York.

Coleman, J.S., 1964: Introduction to Mathematical Sociology. New York.

Coleman, J.S., und McRae, D., 1960: Electronic Processing of Sociometric Data for Groups up to 1000 in Size. American Sociological Review 25.

Coleman, J.S., Katz, E., und Menzel, H., 1966: Medical Innovation. A Diffusion Study. Indianapolis.

Festinger, L., 1949: The Analysis of Sociograms Using Matrix Algebra. Human Relations 2.

Flament, C., 1963: Applications of Graph Theory to Group Structure. Englewood Cliffs.

Forsyth, E., und Katz, L., 1946: A Matrix Approach to the Analysis of Sociometric Data. Sociometry 9.

Gleason, T.C., 1969: Multidimensional Scaling of Sociometric Data. Dissertation. University of Michigan, Ann Arbor.

Harary, F., und Ross, I.C., 1957: A Procedure for Clique Detection Using the Group Matrix. Sociometry 20.

Harary, F., Norman, R.Z., und Cartwright, D., 1965: Structural Models. An Introduction to the Theory of Directed Graphs. New York.

Hoivik, T., und Gleditsch, N.P., 1970: Structural Parameters of Graphs: A Theoretical Investigation. Quality and Quantity 4.

Hummell, H.J., 1972: Probleme der Mehrebenenanalyse. Stuttgart.

Kappelhoff, P., 1976: Die Bestimmung und Charakterisierung primärer Umwelten mit Hilfe soziometrischer Daten im Rahmen eines Mehrebenenmodells. Diss. Köln.

Langeheine, R., 1976: Measures of Social Proximity and their Use in Sociometric Research. With a Computer Program. Unveröffentlichtes Manuskript. Universität Kiel.

Luce, R.D., 1950: Connectivity and Generalized Cliques in Sociometric Group Structure. Psychometrika 15.

Luce, R.D., und Perry, A., 1949: A Method of Matrix Analysis of Group Structure. Psychometrika 14.

McRae, D., 1960: Direct Factor Analysis of Sociometric Data. Sociometry 23.

Michael, J.A., 1961: High School Climates and Plans for Entering College. Public Opinion Quaterly 25.

Peay, E.R., 1975: Nonmetric Grouping: Clusters and Cliques. Psychometrika 40.

Riley, M.W., 1963: Sociological Research. A Case Approach, I. New York.

Scheuch, E.K., 1967: Entwicklungsrichtungen bei der Analyse sozialwissenschaftlicher Daten. In: König, R. (Hrsg.): Handbuch der empirischen Sozialforschung, Bd. I. Stuttgart. 
Weiss, R.S., und Jacobson, E., 1955: A Method for the Analysis of the Structure of Complex Organizations, American Sociological Review 20.
Wössner, J., 1969: Das soziale Feld - Versuch zu einer soziologischen Grundkategorie. Kölner Zeitschrift für Soziologie und Sozialpsychologie 21. 